\title{
Radial rescaling approach for the eigenvalue problem of a particle in an arbitrarily shaped box
}

\author{
Erwin Lijnen, Liviu F. Chibotaru, ${ }^{*}$ and Arnout Ceulemans \\ Department of Chemistry and INPAC Institute for Nanoscale Physics and Chemistry, Katholieke Universiteit Leuven, \\ Celestijnenlaan 200F, B-3001 Leuven, Belgium
}

(Received 4 September 2007; revised manuscript received 26 October 2007; published 3 January 2008)

\begin{abstract}
In the present work we introduce a methodology for solving a quantum billiard with Dirichlet boundary conditions. The procedure starts from the exactly known solutions for the particle in a circular disk, which are subsequently radially rescaled in such a way that they obey the new boundary conditions. In this way one constructs a complete basis set which can be used to obtain the eigenstates and eigenenergies of the corresponding quantum billiard to a high level of precision. Test calculations for several regular polygons show the efficiency of the method which often requires one or two basis functions to describe the lowest eigenstates with high accuracy.
\end{abstract}

DOI: 10.1103/PhysRevE.77.016702

PACS number(s): 02.70.-c, 73.21.La, 73.22.Dj, 73.43.Cd

\section{INTRODUCTION}

Only a few quantum billiards (with Dirichlet boundary conditions) have been found to be exactly solvable. For smooth boundaries, only the circle and the ellipse are known to be solvable [1,2], whereas the family of solvable polygonal quantum billiards is limited to only four cases. They correspond with the three triangles [3-6]: $\Delta(\pi / 2, \pi / 3, \pi / 6)$, $\Delta(\pi / 3, \pi / 3, \pi / 3), \Delta(\pi / 2, \pi / 4, \pi / 4)$, and the general rectangle and inherit their solvability from the fact that their eigenfunctions can be expressed as finite sums over plane waves, a property which is only shared by these four configurations [7]. For all other geometries, only a part of or even none of the eigenfunctions are expressible in analytical form. To solve for these geometries, one usually resorts to purely numerical methods or applies the embedding method $[8,9]$. Although this last method is variational in nature, one is usually obliged to start from trial wave functions which are nonvanishing at the boundary walls. As a result, the formalism dictates that the potential outside the box cannot be taken infinitely large but has to be replaced by a constant but very high potential. This inevitably leads to an error in the eigenvalues, due to leakage of the wave function outside the confined region, which has to be taken into account and corrected for. A further inconvenience is that there are no general guidelines as to whether a chosen basis set will contain sufficient flexibility to satisfy the zero amplitude boundary conditions and therefore lead to good convergence.

In a recent work [10] an alternative approach to solve the particle-in-a-box problem was proposed. It starts from exactly known solutions for the particle in a circular disk, which are subsequently rescaled in a way to obey the desired boundary conditions. In the present article we develop further this idea by giving a procedure for the construction of such basis functions obeying Dirichlet boundary conditions for arbitrarily shaped two-dimensional samples and by setting up practical calculations with them. The scala of quantum billiards which can be treated in this way is quite large and encompasses, for instance, all convex billiards. A more

\footnotetext{
*Liviu.Chibotaru@chem.kuleuven.be
}

complete description of all treatable cases will be given after the actual rescaling procedure has been discussed.

The general setup of the paper is as follows: first we quickly discuss the solutions for a circular disk and thoroughly explain the fundamentals of our coordinate transformation which leads to a complete basis set for the corresponding quantum billiard. Subsequently, we consider some of the subtleties involved when solving for a polygonal billiard. In this case, the rescaled basis functions will show a nonanalytical behavior (due to the corners of the polygonal boundary) which must carefully be taken into account when calculating matrix elements involving these functions. Finally, we derive a general methodology and apply it to the set of regular $n$-gons, with $n$ ranging from 3 to 6 . A short discussion of their resulting eigenvalue spectra and a comparison with previously obtained results $[11,12]$ is also given

The current exposition is obviously not only of academic interest. In fact, the present article originated from a demand for a theoretical interpretation of local density of state (LDOS) measurements performed with low temperature (LT) ultrahigh vacuum (UHV) scanning tunneling microscopy (STM) on nanoscale double-layer cobalt islands deposited on an atomically flat $\mathrm{Au}(111)$ surface [13]. At the simplest level of theory these LDOS maps can be simulated using a particle-in-a-box (quantum billiard) approach, where the surface-state electrons are confined by an infinite potential (Dirichlet boundary conditions) near the edges of the deposited island [14]. As the dimensions of the islands are very small, only a few $\mathrm{nm}$ in diameter, the energy separation between consecutive eigenstates will be quite large and the experimentally obtained LDOS images can be described very accurately as superpositions of only the lowest-lying eigenstates. In a recent article [13], we accurately simulated the LDOS images of such cobalt islands using the rescaling method proposed in this article.

Applications are however not limited to this special case of nanoscaled 2D semiconductor islands but apply to all other sorts of quantum dots, eigenmodes of a vibrating membrane, the resonant modes of an oscillating electromagnetic field in a resonant cavity [15], etc. Note that the current exposition would not be complete without referring to the field of quantum chaos, where the study of quantum billiards also plays a central role $[16,17]$. In quantum chaos one is 
very much interested in the statistical properties of the eigenstates and eigenenergies which involves calculating thousands of eigenvalues and eigenfunctions. However, in the present article the emphasis lies on the accurate determination of the lowest eigenstates and eigenenergies to a very high precision.

\section{PARTICLE IN A CIRCULAR DISK}

In a mathematical formulation, the solutions of a quantum billiard with Dirichlet boundary conditions correspond to the solutions of the two-dimensional Helmholtz equation

$$
\nabla^{2} \psi(\vec{r})=\lambda \psi(\vec{r})
$$

subjected to the condition that

$$
\psi(\vec{r})=0, \quad \forall \vec{r} \in \partial B,
$$

where $\partial B$ denotes the billiards boundary. Expressed in polar coordinates the Helmholtz equation takes on the form

$$
\hat{H} \psi(r, \varphi)=-\frac{\hbar^{2}}{2 m}\left(\frac{\partial^{2}}{\partial r^{2}}+\frac{1}{r} \frac{\partial}{\partial r}+\frac{1}{r^{2}} \frac{\partial^{2}}{\partial \varphi^{2}}\right) \psi(r, \varphi)=E \psi(r, \varphi),
$$

which, free of any boundary conditions, leads to the separable solutions

$$
\psi(r, \varphi)=R_{l}(r) \Phi_{l}(\varphi)=\frac{1}{\sqrt{2 \pi}} J_{l}(r) e^{i l \varphi} .
$$

Here $l$ denotes the familiar integral valued angular momentum quantum number $(1=0, \pm 1, \pm 2, \pm 3, \ldots)$ and the radial function $J_{l}(r)$ corresponds to an $l$ th order Bessel function of the first kind [18].

Solutions to the Dirichlet problem with circular boundary conditions, with radius equal to $a$, can easily be derived by a linear rescaling $r \rightarrow z_{(l, n)} r / a$ of the radial coordinate such that the $n$th zero of the $l$ th Bessel function $z_{(l, n)}$ coincides with the boundary. In other words, one takes the part of the lth Bessel function up to the $n$th radial node and confines it to the disk. After renormalization these solutions form a complete set of orthonormal eigenfunctions which can be uniquely labeled by their corresponding angular $l$ and radial $n$ quantum numbers:

$$
\psi_{(l, n)}^{\circ}=N_{l, n} J_{l}\left(\frac{z_{(l, n)} r}{a}\right) e^{i l \varphi}=J_{l, n}(r) e^{i l \varphi}
$$

The corresponding energy eigenvalues are given by

$$
E_{(l, n)}^{\circ}=\frac{\hbar^{2} z_{(l, n)}^{2}}{2 m a^{2}}
$$

and because $J_{-l}(r)=(-1)^{l} J_{l}(r)$ the energy spectrum for the disk will be completely characterized by double degeneracies except for the special case of zero angular momentum eigenstates.

\section{BASIS SET CONSTRUCTION}

To obtain a basis set for an arbitrarily shaped billiard, we can now define a coordinate transformation which deforms

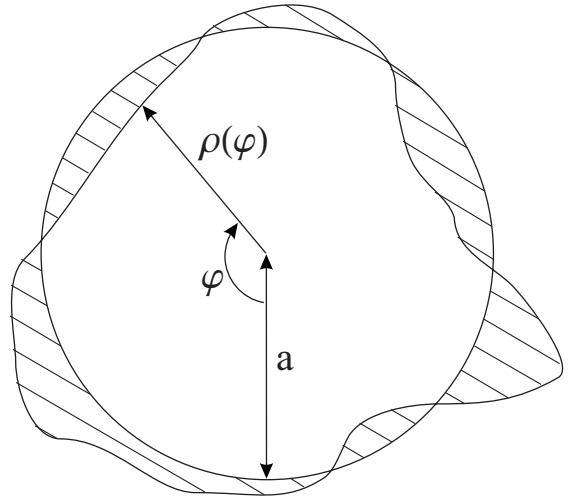

FIG. 1. Deformation of a circular billiard to an arbitrary shaped billiard.

the set of circular disk solutions $\psi_{(l, n)}^{\circ}$ into a set of rescaled functions $\psi_{(l, n)}$ obeying the newly defined boundary conditions [10]. Although in general one could rescale both the radial and angular coordinates, we limit ourselves to the purely radial rescaling

$$
r \rightarrow r^{\prime}=f(r, \varphi), \quad \varphi \rightarrow \varphi^{\prime}=\varphi .
$$

If the origins of the circle $(r=0)$ and the billiard $\left(r^{\prime}=0\right)$ are made to coincide, the new boundary conditions can be formulated as

$$
\psi_{(l, n)}\left(r^{\prime}, \varphi\right)=0 \quad \text { for } \quad r^{\prime}=\rho(\varphi),
$$

where $\rho(\varphi)$ describes the angle-dependent distance of the boundary to the origin (Fig. 1). To reflect the new boundary conditions, the rescaling function $r^{\prime}=f(r, \varphi)$ must thus comply with the following two conditions [10]:

$$
r \rightarrow 0 \Rightarrow r^{\prime} \rightarrow 0, \quad r \rightarrow a \Rightarrow r^{\prime} \rightarrow \rho(\varphi) .
$$

The transformed and the original functions are now simply related by the expression

$$
\psi_{(l, n)}\left(r^{\prime}, \varphi\right)=\psi_{(l, n)}^{\circ}(r, \varphi) .
$$

During the rescaling process, the orthonormality of the new wave functions $\psi_{(l, n)}\left(r^{\prime}, \varphi\right)$ in the region $r^{\prime} \leq \rho(\varphi)$ will generally be lost, but it can be restored by multiplying them by a common normalization function $N$ the exact functional form of which can be retrieved from the equation

$$
\int_{0}^{2 \pi} \int_{0}^{\rho(\varphi)}|N|^{2}\left|\psi_{(l, n)}\left(r^{\prime}, \varphi\right)\right|^{2} r^{\prime} d r^{\prime} d \varphi=1,
$$

which, after the back transformation $r^{\prime} \rightarrow r$ of the radial coordinate, gives

$$
\int_{0}^{2 \pi} \int_{0}^{a}|N|^{2}\left|\psi_{(l, n)}^{\circ}(r, \varphi)\right|^{2}\left(r^{\prime} / r\right)\left(\partial r^{\prime} / \partial r\right) r d r d \varphi=1
$$

Together with the orthonormality of the original disk solutions $\psi_{(l, n)}^{\circ}$ this leads to the following expression for $N$ : 


$$
N=\frac{1}{\sqrt{\left(\partial r^{\prime} / \partial r\right)\left(r^{\prime} / r\right)}} .
$$

To prove that the orthonormal set $N \psi_{(l, n)}\left(r^{\prime}, \varphi\right)$ forms a complete Hilbert space for our boundary problem, we note that its elements are all eigenstates of the Hamiltonian [10]

$$
\hat{H}^{\prime}=N \tilde{H}\left(r^{\prime}, \varphi\right) \frac{1}{N},
$$

where $\tilde{H}\left(r^{\prime}, \varphi\right)$ implies the coordinate transformation of Eq. (7) within $\hat{H}(r, \varphi)$. Notice that in some instances it could be cumbersome, if not impossible, to obtain a closed form expression for the normalization function $N$. However, as this function is common to all rescaled basis functions it can be left out without harming the completeness of the basis set, although this generally means one has to sacrifice orthogonality. A general proof of this last statement is quite straightforward. Due to the completeness of the set $N \psi_{(l, n)}\left(r^{\prime}, \varphi\right)$, any function $f\left(r^{\prime}, \varphi\right)$ obeying the Dirichlet boundary conditions can be expanded in this basis set. As a multiplication of this function with the normalization factor $N$ will retain the boundary conditions, the product function $N f\left(r^{\prime}, \varphi\right)$ can similarly be expanded in this basis set:

$$
N f\left(r^{\prime}, \varphi\right)=\sum_{n, l} c_{n l} N \psi_{(l, n)}\left(r^{\prime}, \varphi\right)
$$

After multiplication of both sides with $N^{-1}$ we obtain

$$
f\left(r^{\prime}, \varphi\right)=\sum_{n, l} c_{n l} \psi_{(l, n)}\left(r^{\prime}, \varphi\right)
$$

which proves that each function $f\left(r^{\prime}, \varphi\right)$ can be expanded in terms of the functions $\psi_{(l, n)}\left(r^{\prime}, \varphi\right)$, which therefore indeed form a complete basis.

\section{A. Radial rescaling}

Before we can put our methodology into practice, we need to specify the functional form of the radial rescaling $r^{\prime}=f(r, \varphi)$. In addition to the constraints of Eq. (9) we further impose that the rescaled functions are well behaved (continuous first derivatives) in the whole region enclosed by the boundary.

The easiest trial function for such a rescaling would be a simple linear deformation of the form [10]

$$
r^{\prime}=\frac{\rho(\varphi)}{a} r
$$

Although it exactly obeys the constraints of Eq. (9) it introduces some problems around the origin which become clear if one takes a closer look at the general form of the Bessel functions $J_{l}(r)$ of positive integer order

$$
J_{l}(r)=\sum_{p=0}^{\infty} \frac{(-1)^{p}}{p !(p+l) !}\left(\frac{r}{2}\right)^{2 p+l} .
$$

Applying the linear transformation of Eq. (17) to Eq. (18) we find the exact form of the rescaled Bessel functions to be

$$
J_{l}\left(r^{\prime}\right)=\sum_{p=0}^{\infty} \frac{(-1)^{p}}{p !(p+l) !}\left(\frac{a r^{\prime}}{2 \rho(\varphi)}\right)^{2 p+l} .
$$

It is easily checked that these rescaled functions are continuous at the origin, but the same cannot be said about their first radial derivatives. In fact, close to the origin the radial derivative of the rescaled first order Bessel function $J_{1}\left(r^{\prime}\right)$ becomes

$$
\frac{\partial J_{1}\left(r^{\prime}\right)}{\partial r^{\prime}} \rightarrow \frac{a}{2 \rho(\varphi)},
$$

which clearly shows angular dependence. To be wellbehaved at the origin, this first derivative should be continuous when passing straight through the origin (from direction $\varphi$ to $\varphi+\pi)$. This condition will, however, only be met for the special case of centrosymmetric billiards when Eq. (20) becomes an even function. For all other geometries, the simple linear trial function of Eq. (17) will certainly lead to discontinuity problems at the origin. Note that we especially investigated the first order Bessel function as it is the only Bessel function which has a linear term in $r^{\prime}$ and therefore the only one which can have a nonzero first radial derivative at the origin.

To remove the discontinuity at the origin, we must search for a more intricate rescaling which can at least limit the influence of the boundary geometry to second or even higher radial derivatives. One such solution is to perform a $d$ th order polynomial rescaling of the form

$$
r=r^{\prime}+\left(\frac{r^{\prime}}{\rho(\varphi)}\right)^{d}[a-\rho(\varphi)], \quad \text { with } d>1
$$

It can be readily checked that this expression satisfies the conditions of Eq. (9). Furthermore, as the angular dependence is moved to the nonlinear term, the rescaled functions will at least be continuous up to $d$ th order radial derivatives. This together with the restraint $d>1$ implies that the first radial derivative will certainly be well behaved.

The behavior of the angular derivative is easier to investigate as it is simply related to the boundary function $\rho(\varphi)$. In fact, the rescaled functions will be well behaved if and only if the first angular derivative of the boundary function $\rho(\varphi)$ is continuous, i.e., if the geometry of the treated quantum billiard is free of any cusps (corners).

In general our methodology thus applies to all cuspless quantum billiards for which there exist a central point from which all boundary points can be reached by the use of straight lines, i.e., if the boundary function $\rho(\varphi)$ can be written as a single-valued function. Clearly, polygonal quantum billiards do not satisfy this condition, but as we shall see in the next section, they can be treated on a similar basis if one only carefully accounts for the nonanalytical character of their resulting basis functions.

\section{POLYGONAL BILLIARDS}

To briefly sketch the main problem related to polygonal quantum billiards, in Fig. 2 we show the radially rescaled basis function $\psi_{(0,1)}$ for the square obtained by applying the 


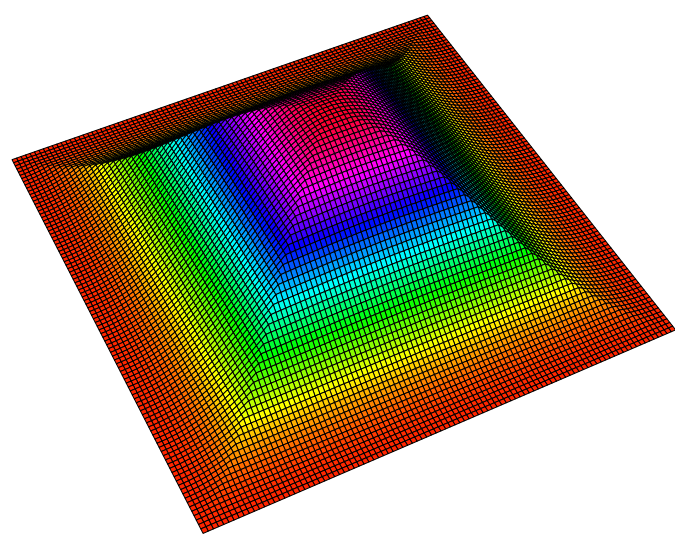

FIG. 2. (Color online) The rescaled basis function $\psi_{(0,1)}$ for the square obtained by applying the radial rescaling of Eq. (21) with $\mathrm{d}=1.2$

rescaling of Eq. (21) to the ground-state function $\psi_{(0,1)}^{\circ}$ of the circular disk. It can clearly be seen that the total function is no longer well behaved as each corner in the boundary geometry introduces an angular discontinuity line ranging from the bordering cusp to the origin. Between two cusps, however, the boundary function $\rho(\varphi)$ and its corresponding rescaled function are both well behaved. Subsequent cusps on the polygonal boundary therefore lead to a natural separation of the polygonal billiard into well-behaved triangular segments which can be treated independently.

As we will be using a variational approach to obtain the lowest eigenvalues and eigenfunctions of our billiard, we must be able to correctly calculate the overlap and energy matrix elements between any set of rescaled basis functions. As the function value itself is continuous everywhere, the overlap integral between two rescaled functions can just be treated as a sum of the overlap integrals for the independent triangles

$$
\left\langle\psi_{(p, q)} \mid \psi_{(s, t)}\right\rangle=\sum_{i}\left\langle\psi_{(p, q)} \mid \psi_{(s, t)}\right\rangle_{\delta_{i}},
$$

where the subscript $\delta_{i}$ indicates an integration over the $i$ th segment.

The energy matrix elements can be calculated in a similar way although one should now also correct for the discontinuity lines between them:

$$
\left\langle\psi_{(p, q)}|\hat{H}| \psi_{(s, t)}\right\rangle=\sum_{i}\left\langle\psi_{(p, q)}|\hat{H}| \psi_{(s, t)}\right\rangle_{\delta_{i}}+\sum_{i} \Delta_{i} .
$$

The contribution $\Delta_{i}$ of a discontinuity line can be seen as the energy matrix element for the shaded surface area (Fig. 3) in the limiting case when the area reduces to a single straight line

$$
\Delta_{i}=\lim _{\varphi_{ \pm} \rightarrow \varphi_{i}} \int_{\varphi_{-}}^{\varphi_{+}} \int_{0}^{\widetilde{\rho}(\varphi)} \psi_{(p, q)}^{\star} \hat{H} \psi_{(s, t)} r^{\prime} d r^{\prime} d \varphi
$$

with

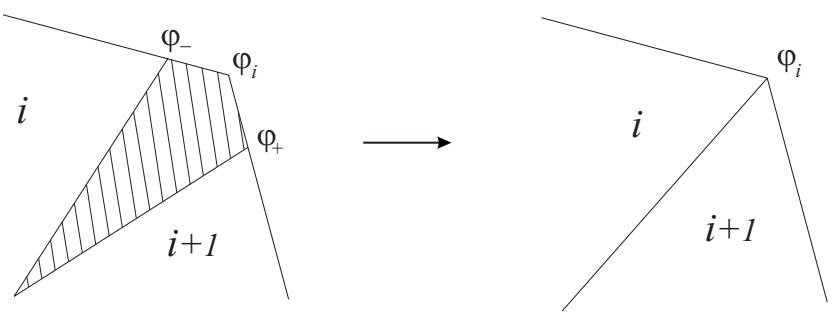

FIG. 3. The contribution of a discontinuity line can be calculated as the energy matrix element of the shaded area in the limiting case when it collapses to a single straight line, i.e., by a line integral.

$$
\tilde{\rho}(\varphi)= \begin{cases}\rho_{i}(\varphi), & \varphi \leq \varphi_{i}, \\ \rho_{i+1}(\varphi), & \varphi \geq \varphi_{i},\end{cases}
$$

where $\rho_{i}(\varphi)$ and $\rho_{i+1}(\varphi)$ denote the boundary functions for the segments separated by the $i$ th discontinuity line.

It will be helpful to further split the Hamiltonian operator into two parts $\hat{H}_{r^{\prime}}$ and $\hat{H}_{\varphi}$, respectively, containing only radial and angular derivatives

$$
\hat{H}\left(r^{\prime}, \varphi\right)=\left(\frac{\partial^{2}}{\partial r^{\prime 2}}+\frac{1}{r^{\prime}} \frac{\partial}{\partial r^{\prime}}\right)+\left(\frac{1}{r^{\prime 2}} \frac{\partial^{2}}{\partial \varphi^{2}}\right)=\hat{H}_{r^{\prime}}+\hat{H}_{\varphi},
$$

where for simplicity the common factor $-\frac{\hbar^{2}}{2 m}$ has been omitted. Based on this separation, the line correction of Eq. (24) splits into two independent parts

$$
\begin{gathered}
\Delta_{i}=\Delta_{i}^{r^{\prime}}+\Delta_{i}^{\varphi}, \\
\Delta_{i}^{r^{\prime}}=\lim _{\varphi_{ \pm} \rightarrow \varphi_{i}} \int_{\varphi_{-}}^{\varphi_{+}} d \varphi \int_{0}^{\widetilde{\rho}(\varphi)} \psi_{(p, q)}^{\star} H_{r^{\prime}} \psi_{(s, t)} r^{\prime} d r^{\prime}, \\
\Delta_{i}^{\varphi}=\lim _{\varphi_{ \pm} \rightarrow \varphi_{i}} \int_{\varphi_{-}}^{\varphi_{+}} d \varphi \int_{0}^{\widetilde{\rho}(\varphi)} \psi_{(p, q)}^{\star} \hat{H}_{\varphi} \psi_{(s, t)} r^{\prime} d r^{\prime} .
\end{gathered}
$$

Closer investigation shows that the radial part $\Delta_{i}^{r^{\prime}}=0$ and therefore does not contribute to the total line correction. The angular part, however, does contribute and can be rewritten in the form

$$
\Delta_{i}^{\varphi}=\lim _{\varphi_{ \pm} \rightarrow \varphi_{i}} \int_{\varphi_{-}}^{\varphi_{+}} \int_{0}^{\widetilde{\rho}(\varphi)} \psi_{(p, q)}^{\star} d \varphi \frac{\partial^{2}}{\partial \varphi^{2}} \psi_{(s, t)} \frac{1}{r^{\prime}} d r^{\prime}
$$

which readily reduces to the line integral:

$$
\Delta_{i}^{\varphi}=\int_{0}^{\rho\left(\varphi_{i}\right)} \psi_{(p, q)}^{\star}\left[\psi_{(s, t)}^{(\varphi)}\left(r^{\prime}, \rho_{i+1}\left(\varphi_{i}\right)\right)-\psi_{(s, t)}^{(\varphi)}\left(r^{\prime}, \rho_{i}\left(\varphi_{i}\right)\right)\right] \frac{1}{r^{\prime}} d r^{\prime} .
$$

Here the terms $\psi_{(s, t)}^{(\varphi)}\left(r^{\prime}, \rho_{i}\left(\varphi_{i}\right)\right)$ and $\psi_{(s, t)}^{(\varphi)}\left(r^{\prime}, \rho_{i+1}\left(\varphi_{i}\right)\right)$ denote, respectively, the left and right first angular derivatives of the rescaled function $\psi_{(s, t)}$ at the $i$ th discontinuity line.

We can thus conclude that our rescaling method can even be applied to polygonal billiards if only one treats the effect 

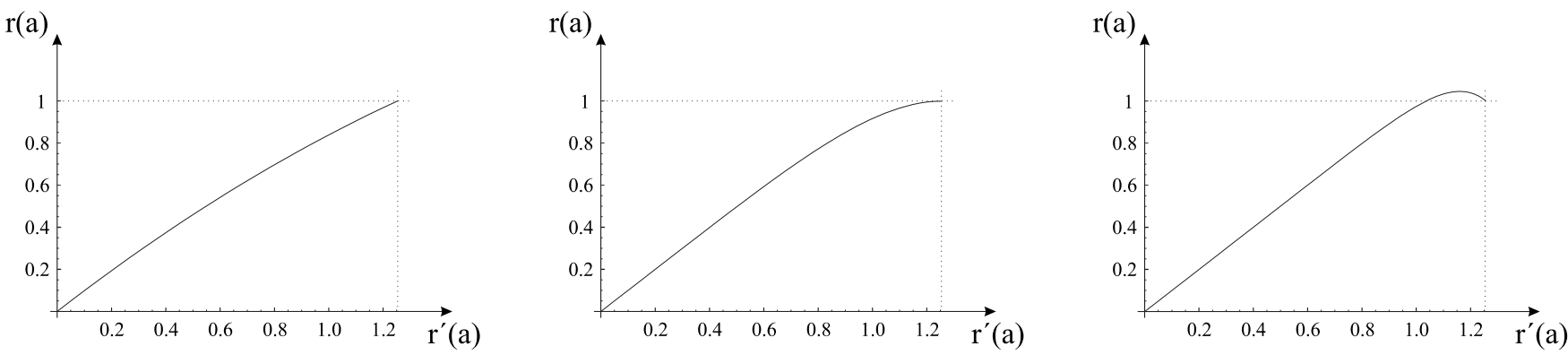

FIG. 4. Plots of the rescaling function (21) for the square using different values of $d(\varphi=\pi / 4)$. (Left) $d<d_{\text {sp }}$, (middle) $d=d_{\mathrm{sp}}$, (right) $d>d_{\mathrm{sp}}$, where $d_{\mathrm{sp}}$ is defined in Eq. (42).

of each discontinuity line by its corresponding line integral. The set of treatable quantum billiards is thus considerably enlarged and now also encompasses those billiards for which the single-valued boundary function $\rho(\varphi)$ has a finite amount of cusps. In the next section, we will describe a general methodology which can be applied to find the eigenenergies and eigenfunctions of all these billiards.

\section{GENERAL METHODOLOGY}

A first and crucial step is the determination of the size and position of the initial circular disk solutions. To get the most out of the current methodology, we want to keep the boundary function $\rho(\varphi)$ of the investigated quantum billiard as close as possible to the radius of the original circular disk. It is therefore natural to start from circular disk solutions which have the same surface area as the quantum billiard and to maximize their mutual overlap (minimize the shaded part of Fig. 1). This, of course, is always subject to the restriction that the boundary function $\rho(\varphi)$ can be written as a singlevalued function. For the special subclass of convex billiards this simply means that the origin of the circle is best pinpointed at the quantum billiards center-of-mass.

The next step consists in determining the degree $d$ of the radial rescaling (21). The lower bound on $d(d>1)$ was already discussed and was needed for the rescaled function to be well behaved around the origin. The upper bound is less trivial and arises from the condition that the rescaling function (21) must be monotonically increasing in the region $r^{\prime}$ $\leq \rho(\varphi)$ and this for all values of $\varphi$. For $\rho(\varphi)<a$ we should not expect any problems as both terms in Eq. (21) will be strictly increasing. For $\rho(\varphi)>a$, however, the behavior is more intricate as for high values of $d$ the rescaling function can become nonmonotonic (Fig. 4). In this last scenario, some values of $r^{\prime}$ will correspond with $r$ values larger then the radius $a$ of the circular disk. Clearly this must be avoided as it would map parts of the circular disk solutions $\psi_{l, n}^{\circ}$, which lie outside the circular boundary, into the bounded region of the rescaled functions $\psi_{l, n}$. In order for Eq. (21) to be monotonic, we simply have to impose

$$
\frac{\partial r}{\partial r^{\prime}} \geq 0 \quad \text { or } \quad[\rho(\varphi)-a] \frac{d\left(r^{\prime}\right)^{d-1}}{\rho(\varphi)^{d}} \leq 1
$$

Irrespective of the value of $d$, the left-hand side of the last inequality attains its highest value when $r^{\prime}$ is maximal, i.e., when $r^{\prime}=\rho(\varphi)_{\max }$. Making the substitutions $r^{\prime} \rightarrow \rho(\varphi)_{\max }$ and $\rho(\varphi) \rightarrow \rho(\varphi)_{\max }$, we find the upper bound on $d$ to be

$$
d \leq \frac{1}{1-a / \rho(\varphi)_{\max }} .
$$

In principle, any value of $d$ between the derived bounds would do, but the quality of the resulting basis set (number of basis functions needed for good convergence) is highly sensitive to the particular choice. A good guideline is to choose the value of $d$ such that the energy of the rescaled ground state function $\psi_{(0,1)}$ is minimized. Of course, all other circular functions must be rescaled accordingly in order to produce a complete Hilbert space for the new boundary problem.

Notice that once the exact form of the radial rescaling has been determined, the resulting basis functions still need to be made orthonormal. As discussed earlier, this can be done by multiplying them by a common rescaling function (13). This, however, requires that $r^{\prime}$ can be expressed in function of $r$ which is not necessarily analytically possible for all values of $d$. For this reason, we chose to perform a Löwdin (symmetric) orthonormalization [19]. The main advantage of this orthonormalization process over the standard Gramm-Smidt procedure is that the resulting functions have much higher resemblance with the original basis set, which makes it possible to link the orthonormalized basis functions with their original circular symmetrical parental functions.

\section{REGULAR POLYGONS $(n=3 \rightarrow 6)$}

As a case study, we apply our methodology to some regular polygons, namely, the regular triangle, the square, the regular pentagon and the regular hexagon. In order to compare their corresponding energy eigenvalues, all polygons are chosen such that they cover the same surface area $\pi a^{2}$ as a circular disk with radius $a$. As the solutions of the regular triangle and the square are known analytically, this at the same time allows us to check for the accuracy of our method.

To calculate the matrix elements, the regular $n$-gons are first subdivided into triangular segments (Fig. 5), for which the boundary functions can be written down in closed form 


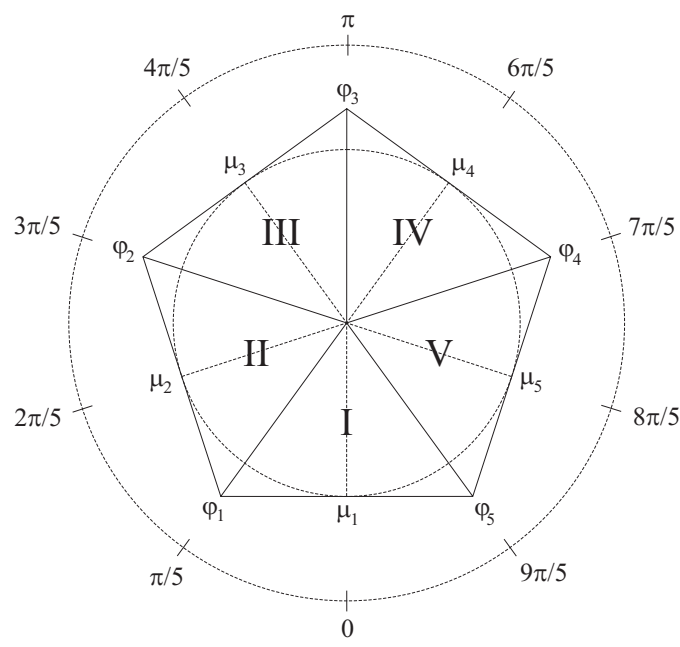

FIG. 5. Segmentation of the regular pentagon into triangles for each of which the boundary function can be written down in closed form (33).

$$
\rho_{i}(\varphi)=\frac{r_{\text {in }}}{\cos \left(\varphi-\mu_{i}\right)} .
$$

Here the value $\mu_{i}$ indicates the angular direction pointing to the middle of the $i$ th side and $r_{\text {in }}$ denotes the radius of the inscribed circle which for a regular $n$-gon with surface area $\pi a^{2}$ equals

$$
r_{\text {in }}=\frac{a \sqrt{\pi}}{\sqrt{n \tan \left(\frac{\pi}{n}\right)}} .
$$

Using the methodology of the previous section, we can now calculate the appropriate eigenvalues and eigenfunctions for a regular $n$-gon. It, however, remains to specify the rescaling order $d$. Although we would normally derive this value through a minimalization of the ground-state energy, in the current case we can adopt a more elegant approach for there exists a special value of $d$ which completely removes the discontinuity lines in the rescaled wave functions.
From Eq. (30) one sees that the $i$ th discontinuity line can only disappear if

$$
\psi_{(s, t)}^{(\varphi)}\left(r^{\prime}, \rho_{i+1}\left(\varphi_{i}\right)\right)-\psi_{(s, t)}^{(\varphi)}\left(r^{\prime}, \rho_{i}\left(\varphi_{i}\right)\right)=0 \quad \text { for } 0<r^{\prime}<\rho_{i}\left(\varphi_{i}\right) .
$$

As all separating lines are symmetry equivalent, it suffices to investigate the above condition for the line in the direction $\varphi=\pi / n$ which after substitution of $\psi_{(s, t)}$ by its explicit functional form gives

$$
\begin{aligned}
& \left.\left(\frac{\partial J_{s, t}(r)}{\partial_{R} \varphi} e^{i l \varphi}+i l J_{s, t}(r) e^{i l \varphi}-\frac{\partial J_{s, t}(r)}{\partial_{L} \varphi} e^{i l \varphi}-i l J_{s, t}(r) e^{i l \varphi}\right)\right|_{\varphi=\pi / n} \\
& \quad=0 .
\end{aligned}
$$

Here $r$ is given by Eq. (21) and $\partial_{R} \varphi$ and $\partial_{L} \varphi$, respectively, denote right and left angular derivatives at the line $\varphi=\pi / n$. In addition to the obvious cancellation of the second and fourth term, the symmetry of the regular polygon further dictates that the first and third term are equal in magnitude (but different in sign), leading to the simpler condition

$$
\left.\frac{\partial J_{s, t}(r)}{\partial \varphi}\right|_{\varphi=\pi / n}=0 \quad \text { for } \quad 0<r^{\prime}<\rho_{i}\left(\varphi_{i}\right) .
$$

Using the expression for the derivative of an $l$ th order Bessel function

$$
\frac{\partial J_{l}(r)}{\partial r}=\frac{1}{2}\left[J_{l-1}(r)-J_{l+1}(r)\right]
$$

together with the chain rule we find

$$
\frac{\partial J_{s, t}(r)}{\partial \varphi} \sim\left[J_{s-1, t}(r)-J_{s+1, t}(r)\right] \frac{\partial r}{\partial \varphi} .
$$

As the first factor, containing the difference of an $(s-1)$ th and $(s+1)$ th order Bessel function, can never be equal to zero for all values of $r^{\prime}$, the equality in Eq. (39) has to arise from the angular derivative of the rescaling function $r$

TABLE I. Energies of the ten lowest eigenstates for the regular polygons $n=3 \rightarrow 6$ and the circle (in units of $\hbar^{2} / 2 m a^{2}$ ). The applied basis sets were build starting from the 67 lowest lying circular disk solutions $\left(E<300 \hbar^{2} / 2 m a^{2}\right)$.

\begin{tabular}{ccccccccc}
\hline \hline \multicolumn{1}{c}{ Triangle } & $\begin{array}{c}\text { Triangle } \\
\text { (Exact) }\end{array}$ & $\begin{array}{c}\text { Triangle } \\
\% \text { error }\end{array}$ & Square & $\begin{array}{c}\text { Square } \\
\text { (Exact) }\end{array}$ & $\begin{array}{c}\text { Square } \\
\% \text { error }\end{array}$ & Pentagon & Hexagon & Circle \\
\hline $7.25936\left(0, A_{1}\right)^{0}$ & 7.25520 & 0.057 & $6.28356\left(0, A_{1}\right)^{0}$ & 6.28319 & 0.006 & $6.02340\left(0, A_{1}\right)^{0}$ & $5.92094\left(0, A_{1}\right)^{0}$ & $5.78319\left(A_{1}\right)(l=0)$ \\
$16.9564(1, E)^{1}$ & 16.9288 & 0.163 & $15.7087(1, E)^{1}$ & 15.7080 & 0.004 & $15.2218\left(1, E_{1}\right)^{1}$ & $15.0078\left(1, E_{1}\right)^{1}$ & $14.6820\left(E_{1}\right)(l=1)$ \\
$16.9564(2, E)^{2}$ & 16.9288 & 0.163 & $15.7087(3, E)^{2}$ & 15.7080 & 0.004 & $15.2218\left(4, E_{1}\right)^{2}$ & $15.0078\left(5, E_{1}\right)^{2}$ & $14.6820\left(E_{1}\right)(l=-1)$ \\
$29.1031\left(0, A_{1}\right)^{5}$ & 29.0208 & 0.284 & $25.1355\left(2, B_{2}\right)^{3,4}$ & 25.1327 & 0.011 & $27.0012\left(2, E_{2}\right)^{3}$ & $26.8713\left(2, E_{2}\right)^{3}$ & $26.3746\left(E_{2}\right)(l=2)$ \\
$31.4931(2, E)^{3}$ & 31.4392 & 0.171 & $31.4201\left(0, A_{1}\right)^{5}$ & 31.4159 & 0.013 & $27.0012\left(3, E_{2}\right)^{4}$ & $26.8713\left(4, E_{2}\right)^{4}$ & $26.3746\left(E_{2}\right)(l=-2)$ \\
$31.4931(1, E)^{4}$ & 31.4392 & 0.171 & $31.4242\left(2, B_{1}\right)^{3,4}$ & 31.4159 & 0.026 & $31.2889\left(0, A_{1}\right)^{5}$ & $31.0384\left(0, A_{1}\right)^{5}$ & $30.4713\left(A_{1}\right)(l=0)$ \\
$46.1206(1, E)^{8}$ & 45.9496 & 0.372 & $40.8669(3, E)^{6}$ & 40.8407 & 0.064 & $42.1989\left(3, E_{2}\right)^{6}$ & $39.4770\left(3, B_{2}\right)^{6}$ & $40.7065\left(E_{3}\right)(l=3)$ \\
$46.1206(2, E)^{9}$ & 45.9496 & 0.372 & $40.8669(1, E)^{7}$ & 40.8407 & 0.064 & $42.1989\left(2, E_{2}\right)^{7}$ & $43.5485\left(3, B_{1}\right)^{7}$ & $40.7065\left(E_{3}\right)(l=-3)$ \\
$50.8411\left(0, A_{2}\right)^{6,7}$ & 50.7864 & 0.108 & $53.5888(1, E)^{8}$ & 53.4071 & 0.340 & $48.9258\left(1, E_{1}\right)^{8}$ & $49.8138\left(1, E_{1}\right)^{8}$ & $49.2185\left(E_{1}\right)(l=1)$ \\
$50.9168\left(0, A_{1}\right)^{6,7}$ & 50.7864 & 0.257 & $53.5888(3, E)^{9}$ & 53.4071 & 0.340 & $48.9258\left(4, E_{1}\right)^{9}$ & $49.8138\left(5, E_{1}\right)^{9}$ & $49.2185\left(E_{1}\right)(l=-1)$ \\
\hline \hline
\end{tabular}


TABLE II. Listing of the eigenenergies (in units of $\hbar^{2} / 2 m a^{2}$ ) for the ground and first excited states of several regular polygons $(n=3 \rightarrow 6)$ obtained by the conformal mapping procedure of Molinari (Ref. [12]) and our rescaling method.

\begin{tabular}{cccccccc}
\hline \hline & \multicolumn{3}{c}{ Ground state } & \multicolumn{4}{c}{ First excited state } \\
No. & Molinari & Present work & Exact & No. & Molinari & Present work & Exact \\
\hline 3 & 7.25816 & 7.25936 & 7.25520 & 3 & 17.2835 & 16.9564 & 16.9288 \\
4 & 6.28340 & 6.28356 & 6.28319 & 4 & 15.7383 & 15.7087 & 15.7080 \\
5 & 6.02217 & 6.02340 & & 5 & 15.2216 & 15.2218 & \\
6 & 5.91742 & 5.92094 & & 6 & 14.9958 & 15.0078 & \\
\hline \hline
\end{tabular}

$$
\left.\frac{\partial}{\partial \varphi}\left[r^{\prime}+\left(\frac{r^{\prime}}{\rho(\varphi)}\right)^{d}[a-\rho(\varphi)]\right]\right|_{\varphi=\pi / n}=0
$$

Substituting $\rho(\varphi)$ by the boundary function $\rho_{1}(\varphi)$ of the first triangular segment we get

$$
\left.\frac{\partial}{\partial \varphi}\left[r^{\prime}+\left(\frac{r^{\prime} \cos (\varphi)}{r_{\text {in }}}\right)^{d}\left(a-\frac{r_{\text {in }}}{\cos (\varphi)}\right)\right]\right|_{\varphi=\pi / n}=0,
$$

which after solving for $d$ gives us the special value

$$
d_{\mathrm{sp}}=\frac{\pi}{\sqrt{\pi}-\sqrt{n \cos \left(\frac{\pi}{n}\right) \sin \left(\frac{\pi}{n}\right)}} .
$$

Using this value, all basis functions of the regular polygon will be completely free of any discontinuity lines. It can be readily seen from Eq. (42) that the value $d_{\mathrm{sp}}$ becomes larger when the number of sides is increased. Consequently, the rescaling function (21) will more and more take the form of a straight line until ultimately, for $n \rightarrow \infty, d_{\text {sp }}$ becomes infinitely large and the rescaling function takes on the form $r$ $=r^{\prime}$, thereby exactly reproducing the circular disk solutions. Quite remarkably, the value of $d_{\mathrm{sp}}$ exactly matches the upper bound on $d$ which can be easily checked by filling in the value of $\rho(\varphi)_{\max }$ into Eq. (32), where $\rho(\varphi)_{\max }$ corresponds to the radius $r_{\text {out }}$ of the circumscribed circle

$$
\rho(\varphi)_{\max }=r_{\text {out }}=\frac{a \sqrt{\pi}}{\sqrt{n \cos \left(\frac{\pi}{n}\right) \sin \left(\frac{\pi}{n}\right)}} .
$$

Now that the rescaling function (with $d=d_{\mathrm{sp}}$ ) is completely defined, we can build appropriate basis sets for each of our regular polygonal quantum billiards. A major advantage of the presented rescaling method is that the resulting basis functions will be symmetry adapted in all cases. Therefore, each rescaled basis function can be appointed to one of the irreducible representations of the symmetry group $C_{n v}$ of the regular polygon and symmetry dictates that only matrix elements between functions belonging to the same irreducible representation can be nonzero [21]. Within cyclic $C_{n v}$ symmetry this means that only basis functions for which $p \bmod n=s \bmod n$, with $p$ and $s$ their respective azimuthal quantum numbers, can interact. The total energy-matrix can thus be rewritten in block-diagonal form with each block corresponding to one irreducible representation.

In Table I we give a general overview of the eigenenergies (in units $\hbar^{2} / 2 m a^{2}$ ) of the ten lowest eigenstates for all regular polygons up to the hexagon. For all cases, the utilized

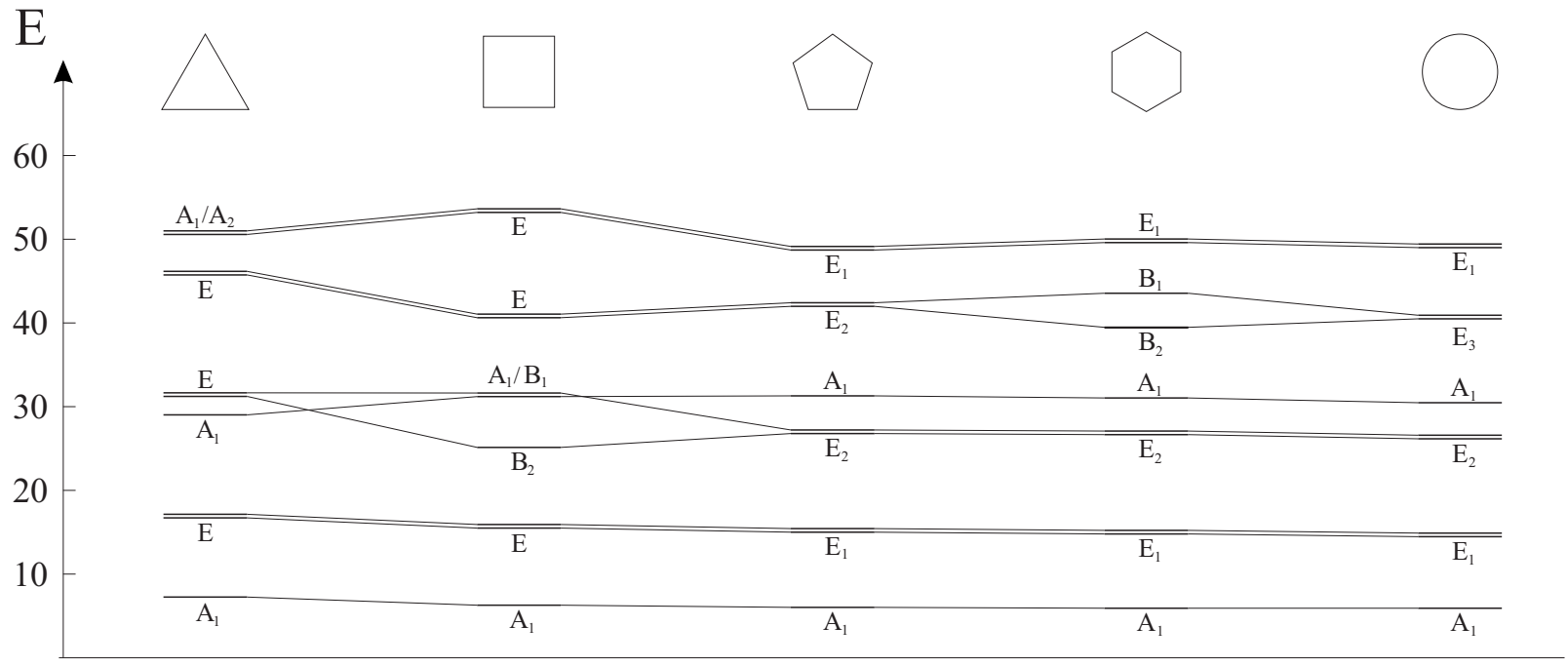

FIG. 6. Correlation diagram for the ten lowest eigenstates of the regular triangle, square, regular pentagon, regular hexagon, and the circle. 

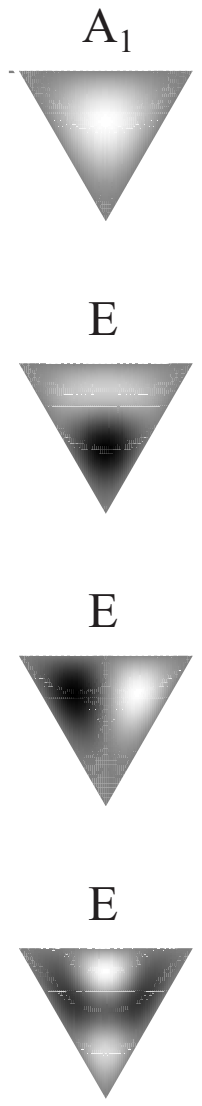

$\mathrm{E}$

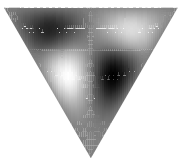

$\mathrm{A}_{1}$

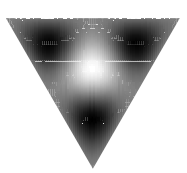

$\mathrm{A}_{1}$

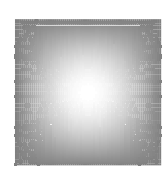

$\mathrm{E}$

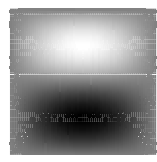

$\mathrm{E}$

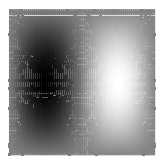

$\mathrm{B}_{1}$
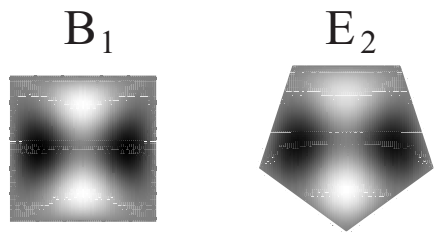

$\mathrm{B}_{2}$
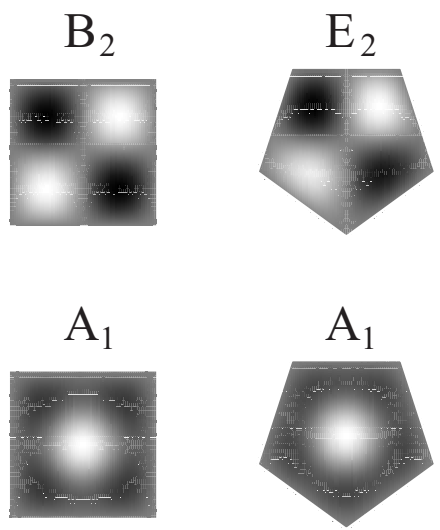
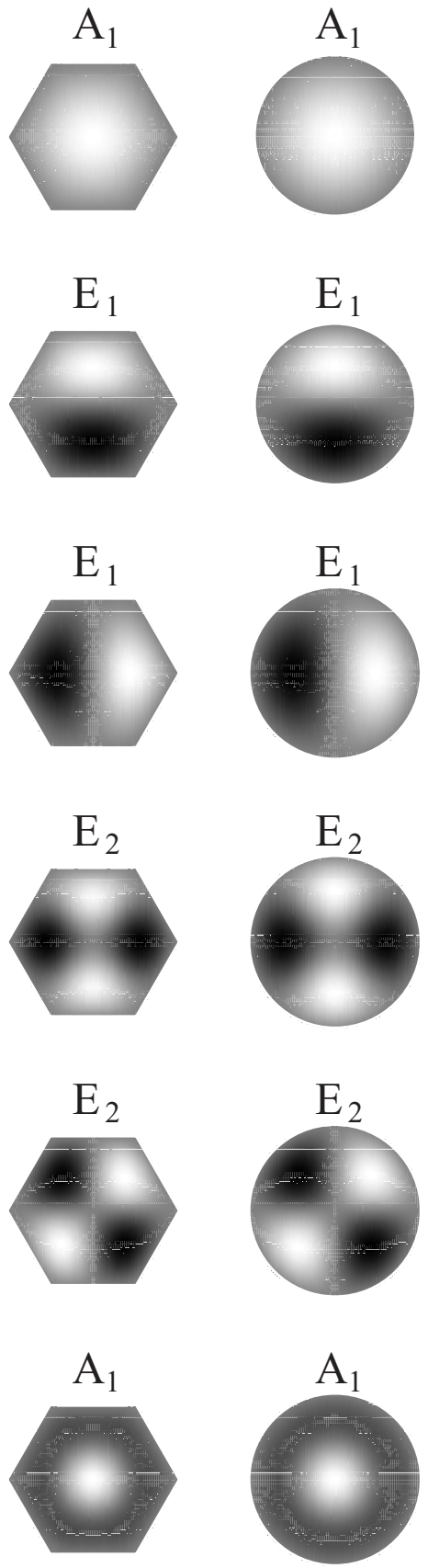

FIG. 7. Plots of the six lowest eigenfunctions for regular triangle, square, regular pentagon, regular hexagon, and the circle corresponding with the numerical results of Table I. Correlated states are listed on the same line.

basis set was built from the 67 lowest lying circular disk solutions (=all solutions up to $E=300 \hbar^{2} / 2 m a^{2}$ ). Next to each calculated value one finds, respectively, the modular class number and the symmetry label of the corresponding eigenstate under the symmetry group $C_{n v}$. To check for the accuracy of our method, the calculated eigenvalues of the triangle and the square were compared with their exactly known values. We observe that, although a fairly moderately sized basis set was used, the results are quite accurate with a fault margin of only a few tenths of a percent. Closer inspection also shows that more accurate results are obtained for the square then for the triangle, which can be attributed to its smaller geometrical deviation from the circle. As a rule of thumb we can state that our method becomes more accurate when the deviation from the original circular boundary becomes smaller [=small $\left.\rho(\varphi)_{\max }\right]$. The energy values for the pentagon and hexagon are thus believed to be at least as accurate as those of the square, if not more accurate.

Although exact solutions for the regular pentagon and hexagon are not available, earlier results on the ground-state energies of regular polygonal billiards $[11,12]$ serve as good benchmarks for our rescaling method. In Table II we list the eigenenergies of the ground and first excited states of several regular polygons $(n=3 \rightarrow 6)$ obtained by the conformal mapping procedure of Molinari (Ref. [12]) together with the results obtained by our rescaling method. As one can see, al- 
though we used a very modest basis set (10 up to 25 basis functions) the accuracy of our results is comparable to that of Molinari, especially for the first excited states. Of course, if higher precision is required, the amount of basis functions can always be increased.

One of the most striking features of the eigenvalue spectra of the regular polygons is the multiple occurrences of degeneracies. Most of them can be explained by the complex conjugation of their corresponding irreducible representations. This is the case for all irreducible representation except for those of 0 and $n / 2$ type. For the special cases of the triangle and the square, one also encounters degeneracies in these last two modular classes $\left(A_{1} / A_{2}\right.$ degeneracy at 50.7864 for the triangle, $A_{1} / B_{1}$ degeneracy at 31.4159 for the square), thereby pointing to the existence of some hidden symmetries [20].

A very natural way to relate the eigenstates of different regular polygons is to classify them according to the circular basis function which is dominant in their basis set expansion. In Table I this classification is given by the superscripted numbers 0 to 9 , which indicate to which circular disk solutions (zero to ninth excited state) these eigenfunctions should be linked. Based on these identifications we constructed a correlation diagram which is shown in Fig. 6. Although the eigenvalues of the ground states and doubly degenerate first excited states follow a general decreasing trend when the number of sides is increased, the higher excited states are subjected to more variation and even do not necessarily follow the same ordering as the circular disk solutions. This is especially the case for the triangle and square which, due to their low cyclic symmetry, exhibit a large amount of mixing. Recall that only basis functions belonging to the same irreducible representation (indicated by the number behind the experimental obtained values) can interact. As the smallest polygons are characterized by a small number of modular classes, there will be a substantial amount of mixing thereby possibly leading to some state crossings. Starting from the pentagon, the listed eigenstates follow exactly the ordering of the circular disk solutions but anomalies will certainly be found for the higher excited states.

To conclude our discussion, we show in Fig. 7 pictorial representations of the six lowest eigenstates for the geometries listed in Table I, where correlated states are depicted on the same line. Notice that in case of degenerate eigenstates, symmetric and antisymmetric linear combinations were taken in order to obtain real valued functions.

\section{CONCLUSIONS}

In this paper we introduced a new rescaling procedure to calculate the lowest eigenenergies and eigenfunctions of a generic quantum billiard. The procedure applies to smooth as well as polygonal billiards with only the limitation that the bordering geometry should be expressible as a single-valued function $\rho(\varphi)$. One of its greatest advantages is that the constructed basis functions already comply with the boundary conditions. Consequently, the exact boundary conditions do not have to be imposed by the associated Hamiltonian operator, which therefore only comprises of a kinetic energy term. Simple variational calculations using modest basis sets lead to results which are accurate up to a few tenths of a percent and which automatically converge to the correct limiting values. As the applied rescaling process is quite versatile, it leaves sufficient space for further improvements, for instance, by introducing an angular dependency in the rescaling order $d$. Notice that future use of our methodology is not limited to 2D-confined systems but in principle can be extended to higher dimensional Dirichlet problems for which the bordering conditions are single valued.

\section{ACKNOWLEDGMENT}

Financial support from the Flemish Science Foundation (FWO) is gratefully acknowledged.
[1] R. W. Robinett, Am. J. Phys. 64, 440 (1996).

[2] A. J. S. Traiber, A. J. Fendrik, and M. Bernath, J. Phys. A 22, L365 (1989).

[3] A. Theophilou and P. Tsilimigras, Nukleonik 12, 280 (1969).

[4] H. R. Krishnamurthy, H. S. Mani, and H. C. Verma, J. Phys. A 15, 2131 (1982).

[5] W.-K. Li and S. M. Blinder, J. Math. Phys. 26, 2784 (1985).

[6] W.-K. Li, J. Chem. Educ. 61, 1034 (1984).

[7] R. L. Liboff, J. Math. Phys. 35, 596 (1994).

[8] S. Crampin, M. Nekovee, and J. E. Inglesfield, Phys. Rev. B 51, 7318 (1995).

[9] D. L. Kaufman, I. Kosztin, and K. Schulten, Am. J. Phys. 67, 133 (1999).

[10] L. F. Chibotaru, A. Ceulemans, M. Lorenzini, and V. V. Moshchalkov, Europhys. Lett. 63, 159 (2003); L. F. Chibotaru, A. Ceulemans, M. Lorenzini, and V. V. Moshchalkov, ibid. 63, 476 (2003).

[11] J. K. Bhattacharjee and K. Banerjee, J. Phys. A 20, L759 (1987)
[12] L. Molinari, J. Phys. A 30, 6517 (1997).

[13] K. Schouteden, E. Lijnen, E. Janssens, P. Lievens, C. Van Haesendonck, A. Ceulemans, and L. F. Chibotaru (unpublished).

[14] J. Li, W.-D. Schneider, S. Crampin, and R. Berndt, Surf. Sci. 422, 95 (1999).

[15] J. Stein and H.-J. Stöckmann, Phys. Rev. Lett. 68, 2867 (1992).

[16] M. C. Gutzwiller, Chaos in Classical and Quantum Mechanics (Springer-Verlag, New York, 1990).

[17] S. R. Jain, B. Grémaud, and A. Khare, Phys. Rev. E 66, 016216 (2002).

[18] F. Bowman, An Introduction to Bessel Functions (Dover, New York, 1958).

[19] P.-O. Löwdin, J. Chem. Phys. 18, 365 (1950).

[20] G. B. Shaw, J. Phys. A 7, 1537 (1974).

[21] L. F. Chibotaru, A. Ceulemans, M. Morelle, G. Teniers, C. Carballeira, and V. V. Moshchalkov, J. Math. Phys. 46, 095108 (2005). 